\title{
A Polarized Fourier Transform Infrared Spectrometry Attenuated Total Reflection Study of Bentonite Settled onto Magnetite
}

\author{
Allan Holmgren*, ${ }^{*}$ and Xiaofang Yang ${ }^{\dagger, *}$ \\ Division of Chemistry, Department of Chemical Engineering and Geosciences, Luleå University of Technology, \\ SE-971 87 Luleå, Sweden and Research Center for Eco-Environmental Sciences, Chinese Academy of Sciences, \\ Beijing 100085, China
}

Received: June 3, 2008; Revised Manuscript Received: August 2, 2008

Sodium bentonite in aqueous suspension was allowed to settle onto a layer of magnetite, and the orientation of the bentonite platelets was monitored using the Fourier transform infrared spectrometry-attenuated total reflection technique. This study is the first reported use of polarized IR to study the in situ settling of delaminated bentonite platelets onto a thin layer of magnetite nanoparticles. The experiment was performed at a $\mathrm{pH}$ value ( $\mathrm{pH}$ 5.6) close to the point of zero charge for the edge surfaces of the bentonite platelets in order to possibly enhance the probability for the (001) surface of bentonite to adhere to the positively charged magnetite particles. The order parameter $(S)$ of the platelets was calculated both for the dry film and the film formed during water evaporation. These results were compared with the orientation of bentonite platelets on the internal reflection element ( $\mathrm{ZnSe}$ ) without magnetite particles, a system where the three layer model is valid. During settling of the bentonite, the tilt angle of the normal to the (001) surface of the platelets decreases and reaches a minimum value for the dry film. When the film is still covered by a layer of water, the tilt angles indicate the film to be at least partly built up of so-called card-house structures, implying that the edge surfaces of the platelets are at least partly adhering to the basal (001) surfaces.

\section{Introduction}

Minerals composed of silicon oxide tetrahedrals combined with aluminum oxide octahedrals are very common in nature. Clay minerals belong to these types of structures, which exhibit swelling and nonswelling properties in water depending on the organization of their tetrahedral and octahedral layers. If the silica tetrahedra and the alumina octahedra stick together as one unit, the mineral is nonswelling, as in kaolinite, whereas a structural unit with $\mathrm{Si}-\mathrm{Al}-\mathrm{Si}$ layers forms swelling clays. ${ }^{1,2}$ The structure of bentonite, commonly constituting $\sim 80 \%$ montmorillonite, is shown in Figure 1. The (001) plane, perpendicular to the surface normal of the bentonite platelets is always negatively charged due to atomic substitution in the octahedral sheet, where $\mathrm{Al}(\mathrm{III})$ is substituted for, for example, $\mathrm{Mg}(\mathrm{II})$ ions. The surface of the silica tetrahedra is, therefore, not sensitive to $\mathrm{pH}$ changes since the charge is due to atomic substitution, whereas the edge surface of the platelet, which consists of metal hydroxide groups, is sensitive to this experimental parameter. There are investigations suggesting that the point of zero charge of the edge surfaces is close to $\mathrm{pH} \approx 5,{ }^{3,4}$ whereas the magnetite particles are positively charged up to $\mathrm{pH}=7.9 .5^{5}$ At $\mathrm{pH}$ values lower than 6 , so-called card-house structures may form due to electrostatic attraction between positive and negative charges, implying that the edges of the bentonite platelets may adhere to the 001 surfaces of other platelets. ${ }^{2}$ If these card-house structures are formed, they will certainly affect the dichroic ratio of the $\mathrm{Si}-\mathrm{O}$ vibrations with transition moments perpendicular and parallel to the surface

*Corresponding author: e-mail: allan.r.holmgren@ltu.se; fax: +46(0)920491199; phone: +46(0)920492140.

${ }^{\dagger}$ Luleå University of Technology.

$\$$ Chinese Academy of Sciences.

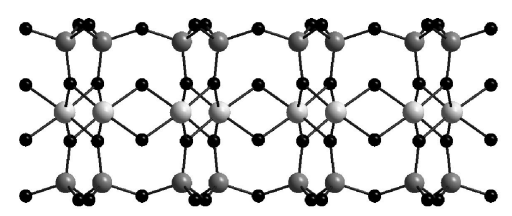

Exchangeable cations \& water molecules

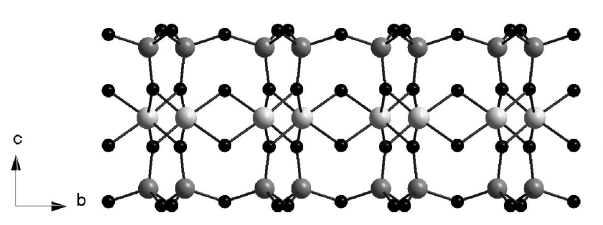

Tetrahedral layer (Si-O)

Octahedral

layer (Me-O)

Tetrahedral layer (Si-O)

Figure 1. The structure of montmorillonite platelets showing the three layers of the platelet viz. the two silica tetrahedra on each side of the alumina or magnesia (Me) octahedra. Between these platelets there are exchangeable cations and water molecules. The ideal composition may be written: $(\mathrm{Na}, \mathrm{Ca})_{1 / 3}\left(\mathrm{Al}_{5 / 3}, \mathrm{Mg}_{1 / 3}\right) \mathrm{Si}_{4} \mathrm{O}_{10}(\mathrm{OH})_{2}$. The thickness of the platelets is $\sim 1 \mathrm{~nm}$, and in the bentonite studied here, a fraction of the Me-atoms are substituted for, e.g., Fe(II) atoms.

normal of the platelets and, consequently, the average orientation of bentonite particles on the particulate magnetite substrate.

Swelling clays imply the possibility of water intercalation between the Si-layers of adjacent structural units, such as in bentonite. The latter types of clays are very popular as adsorbents since they offer the possibility to intercalate anions, cations, and organic molecules such as proteins, in addition to water. Furthermore, the layered structure may cause bentonite to delaminate under shear stress. Because of these properties and because of its abundance in nature, this type of clays has become very popular as adsorbents in a variety of applications, such as in wine purification, as a component in kitty litter, and in water treatment but also as a sealing agent in nuclear waste 
deposition, in geotechnical engineering, and as lubricant in tribology. In the latter application, the layered structure is utilized to reduce friction losses in, for example, oil well drilling. ${ }^{6}$ Bentonite is also frequently used as a binder in pelletizing plants, ${ }^{7-9}$ where it seems to facilitate the agglomeration of valuable mineral particles such as magnetite into mechanically strong green pellets, partly due to its swelling ability. The mechanism by which bentonite acts as a binder in green pellets is not very well-known, although it is reasonable to assume that its negatively charged silicate surfaces and their possibility to adhere to less negatively charged magnetite particles should affect the wetability of the system, and therefore the capillary forces being one of the forces keeping the magnetite particles together. Upon mixing magnetite with bentonite, expanded or delaminated bentonite grains may also form a sort of fibrous structure between the magnetite particles during or before agglomeration.

To obtain information about the orientation of bentonite platelets on magnetite, polarized infrared spectroscopy was a natural choice. Much of the attention in prior and recent IR studies has focused on the behavior of species adsorbed at mineral surfaces, ${ }^{10-16}$ including clay minerals. ${ }^{17-20}$ This technique has also recently been utilized to study the orientation of monolayers of clay minerals on different ATR substrates as well as dye molecules adsorbed on the mineral surface. ${ }^{21-23}$ The clay film was accomplished either by settling from a water suspension or by using the Langmuir-Blodgett method. However, the orientation of clay particles in a thin multilayer of the clay where the first layer is electrostatically interacting with another mineral, such as magnetite, has not been reported so far. This became the challenge of the present study.

This study was, therefore, devoted to the orientation of bentonite particles on the magnetite surface under both wet and dry conditions, since the orientation caused by mineral-metal oxide interaction is believed to be important for the strength of the green pellets, that is, pellets before sintering at elevated temperature. Of special interest was the interaction at very low water contents, where the magnetite-bentonite system is approaching natural dryness. Fourier transform infrared (FTIR) spectroscopy with s-polarized and p-polarized incident radiation in combination with the attenuated total reflection (ATR) technique admitted the orientation of bentonite platelets settled from a water suspension onto magnetite particles to be examined, as well as the orientation of the platelets after a "dry" film had been formed. In this context it might be clarified that settled particles may be regarded as being in aqueous suspension when they are separated by water molecules, but they constitute a settled aqueous suspension as opposed to the bulk suspension.

\section{Materials and Methods}

The Clay Mineral. The clay mineral bentonite, originally from Milos in Greece, was obtained from Minelco and was ion exchanged using $0.6 \mathrm{M} \mathrm{NaCl}$. The bentonite obtained was claimed to be ion exchanged already, so doing it again was rather a precautionary measure. In the exchange procedure, $1 \mathrm{~g}$ of the bentonite was mixed with $50 \mathrm{~mL}$ of the sodium chloride solution, and the suspension was stirred for about $3 \mathrm{~h}$. After stirring, the bentonite settled at the bottom of the beaker because of particle coagulation due to the high sodium chloride concentration, and the clear solution was substituted for another $50 \mathrm{~mL}$ of aqueous sodium chloride. This was repeated three times, and finally the suspension was allowed to settle at elevated temperature $\left(60^{\circ} \mathrm{C}\right)$ for $1 \mathrm{~h}$. The ion-exchanged product was then redispersed in $200 \mathrm{~mL}$ of Milli-Q water $(\mathrm{pH}=5.6)$ under stirring for $8 \mathrm{~h}$. Some larger particles settled rather quickly, whereas the smaller ones were dispersed for days. This $\mathrm{pH}$ was used for reasons explained above, although the $\mathrm{pH}$ used in plants is usually between 8 and 10. The suspension so obtained was then centrifuged at $20000 \mathrm{rpm}$ for $30 \mathrm{~min}$, and this procedure was repeated until the water contained no chloride ions according to an $\mathrm{AgNO}_{3}$ test. These bentonite particles had a BET surface of $53.8 \pm 0.3 \mathrm{~m}^{2} / \mathrm{g}$. The particle size distribution was narrow with peak intensity at $340 \mathrm{~nm}$, as measured by a Zeta Plus instrument from Brookhaven Instruments Corporation. The average size of the particles indicated the clay mineral to be very well-delaminated. The chemical composition of the ionexchanged Milos bentonite in wt \%: $59.6\left(\mathrm{SiO}_{2}\right), 21.9\left(\mathrm{Al}_{2} \mathrm{O}_{3}\right)$, $4.6\left(\mathrm{Fe}_{2} \mathrm{O}_{3}\right), 3.1(\mathrm{MgO}), 3.1\left(\mathrm{Na}_{2} \mathrm{O}\right), 0.5\left(\mathrm{~K}_{2} \mathrm{O}\right), 0.8\left(\mathrm{TiO}_{2}\right), 0.1$ $(\mathrm{CaO}), 0.4$ (Tot $\mathrm{C}$ ). The analyses were performed using inductively coupled plasma-atomic emission spectrometry (ICPAES). ${ }^{24}$

The Magnetite Particles. The synthesis of magnetite was carried out by controlled coprecipitation of $\mathrm{Fe}(\mathrm{II})$ and $\mathrm{Fe}$ (III) when added to an aqueous alkaline solution. ${ }^{25,26} \mathrm{Fe}(\mathrm{II})$ and $\mathrm{Fe}(\mathrm{III})$ chloride solutions were prepared by dissolving the salts in deoxygenated Milli-Q water. A $5 \mathrm{~mL}$ portion of the mixture (molar ratio 1:2; 1.2 $\mathrm{M}$ total iron chloride concentration) was added dropwise $(0.5 \mathrm{~mL} / \mathrm{min})$ to a reaction vessel containing $40 \mathrm{~mL} 0.9 \mathrm{M} \mathrm{NH}_{4} \mathrm{OH}$ solution. The reaction mixture was kept under argon atmosphere at room temperature and was vigorously stirred. After addition of the iron salts, the mixture was stirred for another $20 \mathrm{~min}$, and then the precipitated magnetite was separated from the supernatant by decantation under argon. The solid was washed 4 times with deoxygenated Milli-Q water and separated by decantation. The magnetite particles formed had a diameter of about $20 \mathrm{~nm}$ and were then allowed to settle onto an ATR crystal during evaporation of the dispersion medium (water).

Film Preparation. The magnetite film on the ATR crystal was accomplished by evaporating a freshly synthesized aqueous dispersion of the magnetite particles $(0.15 \mathrm{mg} / \mathrm{mL})$. Because small particles of magnetite have a tendency to oxidize to form hematite or maghemite, especially at elevated temperature, a freshly prepared magnetite suspension was used. The magnetite film was formed during evaporation of the water medium under reduced atmospheric pressure $(\sim 700 \mathrm{mBar})$ and by tilting the length direction of the ATR crystal about $5^{\circ}$. This arrangement allowed the water meniscus to move along the crystal surface and resulted in a smooth and evenly distributed film of the magnetite particles. The magnetite film so formed was allowed to dry in vacuum. Shortly before the IR measurements, 1.25 $\mathrm{mL}$ of a dispersion of bentonite $(0.0125 \mathrm{wt} \%)$ was added to the magnetite film on the ATR crystal. The $\mathrm{pH}$ of this dispersion was measured to be 5.6, in good accordance with $\mathrm{pH}$ of the Milli-Q water used as dispersion medium. The low $\mathrm{pH}$ is due to the proton exchange during ion exchange of the water. Then, the bentonite particles were allowed to settle onto the magnetite surface, and the orientation of the settled platelets were measured in situ by polarized FTIR.

Infrared Spectroscopy. The ATR internal reflection element was a ZnSe crystal plate (New Era Enterprises, Inc.) with the size $80(\mathrm{l}) \times 10(\mathrm{w}) \times 3 \mathrm{~mm}(\mathrm{t})$ and a face angle of $45^{\circ}(13$ reflections on one side, refractive index $=2.41$ ). A horizontal ATR (HATR) trough crystal plate mount was used in these experiments. Infrared spectra were recorded on a Bruker IFS 66v/S FTIR spectrometer equipped with a DTGS detector. All spectra were recorded at room temperature $\left(23^{\circ} \mathrm{C}\right)$ and normal pressure using the double-side forward-backward acquisition mode. The single beam background 


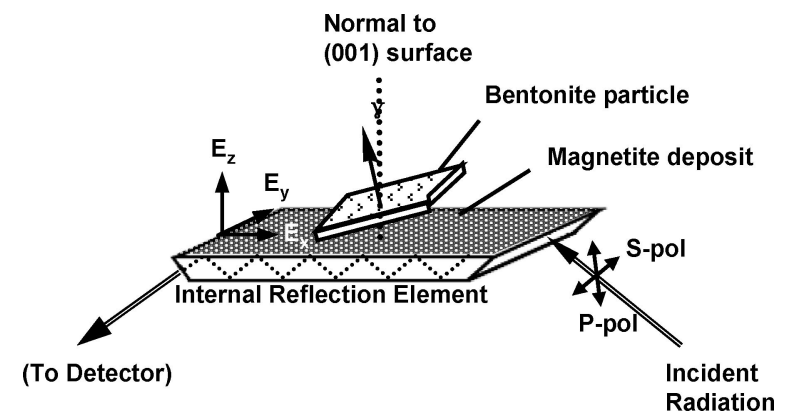

Figure 2. Incident p-polarized and s-polarized radiation is internally reflected through the ATR crystal, producing an evanescent electric field outside the thin magnetite layer at the crystal surface. The dichroic ratio $A_{\mathrm{s}} / A_{\mathrm{p}}$ determines the tilt angle between the normal to the 001 surface of the bentonite platelets and the surface normal of the ATR crystal.

spectrum of $\mathrm{ZnSe}$ with a layer of magnetite was recorded under argon atmosphere. A total number of 256 scans were signalaveraged using an optical resolution of $4 \mathrm{~cm}^{-1}$. The resultant interferogram was Fourier transformed using the Mertz phase correction mode, a Blackman-Harris 3-term apodization function, and a zero-filling factor of 2 .

Polarization of the IR beam was achieved with a rotatable grid polarizer of aluminum deposited on $\mathrm{BaF}_{2}$, placed in front of the HATR accessory. The absorbance of the p-polarized spectra was recorded with the polarizer, allowing the electric field to pass in the plane of the incident radiation $\left(A_{\mathrm{p}}\right)$. In combination with the s-polarized absorbance recorded with the electric field perpendicular to the plane of incidence $\left(A_{\mathrm{s}}\right.$, parallel to the crystal surface), the dichroic ratio $\left(A_{\mathrm{s}} / A_{\mathrm{p}}\right)$ could be calculated.

The Polarized FTIR-ATR Method. The experimental method used here is based on the polarized HATR technique as shown in the following simplified picture (Figure 2). In the model describing the tilt angles of molecules adsorbed on an ATR crystal, a molecular axis is defined. ${ }^{27}$ The tilt angle is the angle between the molecular axis and the surface normal of the ATR crystal and is calculated from the dichroic ratio $\left(A_{\mathrm{s}} / A_{\mathrm{p}}\right)$, assuming that the molecular axis has uniaxially symmetric distribution around the $z$-axis (surface normal of the crystal) and that the transition dipole moments in the molecule have a uniaxially symmetric distribution about the molecular axis. These transition moments form a known angle with the molecular axis. In all-trans alkyl chains, for example, this angle is $\theta=90^{\circ}$ for symmetric and antisymmetric $\mathrm{CH}_{2}$ stretching vibrations. However, there is no such molecular axis in bentonite particles. The molecular axis, in the model used here, is therefore assigned to the (001) surface normal of the bentonite platelets, and the calculated tilt angle is the angle between the surface normal to the platelets and the surface normal to the ATR crystal used $(\mathrm{ZnSe})$.

The measured absorptions ( $A_{\mathrm{s}}$ and $A_{\mathrm{p}}$ ) are proportional to the square of the vector product between the electric field vector of the evanescent field (E) and the transition dipole moment (M). This may be written according to eq 1 ,

$$
A \propto(\mathbf{E} \cdot \mathbf{M})^{2}
$$

where $A$ is the absorbance.

The components of the electric field $(\mathbf{E})$ depends on the refractive indices of the crystal, the layers on the crystal, and the medium outside these layers, in addition to the angle of incidence, which is $45^{\circ}$ in our experimental setup. The equations used to calculate the $x, y$, and $z$ components of the electric field are described by Harrick and Mirabella ${ }^{28,29}$ as well as in a number of publications. ${ }^{23,27,30,31}$ The square of the electric field components are given by eqs $2-4$

$$
\begin{gathered}
E_{x}^{2}=\frac{4 \cos ^{2} \alpha\left(\sin ^{2} \alpha-n_{31}^{2}\right)}{\left(1-n_{31}^{2}\right)\left\{\left(1+n_{31}^{2}\right) \sin ^{2} \alpha-n_{31}^{2}\right\}} \\
E_{y}^{2}=\frac{4 \cos ^{2} \alpha}{1-n_{31}^{2}} \\
E_{z}^{2}=\frac{4 \cos ^{2} \alpha\left(\sin ^{2} \alpha\right) n_{32}^{4}}{\left(1-n_{31}^{2}\right)\left\{\left(1+n_{31}^{2}\right) \sin ^{2} \alpha-n_{31}^{2}\right\}}
\end{gathered}
$$

In eqs $2-4, \alpha$ is the angle of incidence, $n 31=n_{3} / n_{1}$ and $n_{32}$ $=n_{3} / n_{2}$, where $n_{1}$ is the refractive index of the ATR crystal, $n_{2}$ is the refractive index of the inorganic layer (magnetite and bentonite), and $n_{3}$ is the refractive index of the medium outside this layer (water or air). In addition to the angle of incidence of the infrared radiation, the electric field components are described by three refractive indices (the three layer model).

If the second layer is composed of alkyl chain molecules adsorbed onto the ATR crystal, then a molecular axis is commonly defined with transition dipole moments in the molecules forming an angle $\theta$ with respect to the molecular axis. A weakness of this model is that alkyl chains can exhibit trans-gauche conformers, implying that the molecular axis is rather a curve than a straight line. On the other hand, an alltrans conformation of the alkyl chains may imply a biaxial distribution of the molecular axis around the surface normal of the ATR crystal. However, in the present case a uniaxial distribution of transition dipole moments parallel to the (001) surface normal of the bentonite platelets is considered. This model is similar to the one used in polarized infrared transmission spectroscopy of lamellar liquid crystalline phases, except that in the latter experiments the molecular system has to be tilted in order to measure a dichroic ratio. ${ }^{32}$

The average angle between the transition moment and the surface normal of the ATR crystal is probed by the ratio between the absorption of s-polarized and p-polarized infrared radiation and can be calculated by the following equation,

$$
D=\frac{A_{\mathrm{s}}}{A_{\mathrm{p}}}=\frac{E_{y}^{2} \sin ^{2} \gamma}{E_{x}^{2} \sin ^{2} \gamma+2 E_{z}^{2} \cos ^{2} \gamma}
$$

where $\gamma$ is the average tilt angle.

In the derivation of eq 5 , the angle between the transition dipole moment $\mathbf{M}$ and the normal to the (001) surface of bentonite is assumed to be $\theta=0^{\circ}$. Solving eq 5 for $\gamma$ gives eq 6.

$$
\gamma=\arcsin \left(\frac{2 D E_{z}^{2}}{D\left(2 E_{z}^{2}-E_{x}^{2}\right)+E_{y}^{2}}\right)^{1 / 2}
$$

Equation 6 was used to calculate average tilt angles for bentonite platelets settled on the magnetite particles, which were deposited on the $\mathrm{ZnSe}$ internal reflection element.

\section{Results and Discussion}

The theory briefly described above and used in the estimation of the orientation of bentonite platelets on magnetite particles relies on the assumption that the bentonite platelets including the magnetite particles form a thin film on the substrate. This may be accomplished by using a sufficiently diluted suspension of bentonite or by interrupting the settling of the dispersed 
bentonite platelets at an appropriate time. The latter method also serves as an evidence for attractive electrostatic interaction between the thin layer of magnetite particles on the crystal and bentonite platelets settled on this layer. Interrupting the settling process after half an hour and gently washing the crystal with distilled water resulted in only a minor decrease in the measured absorbance of the bentonite film covered with water. A minor decrease might be expected since bentonite platelets still in suspension are now not detected. However, the concentration of bentonite at the surface of the crystal is much higher than in the suspension, at least for diluted suspensions, which explains the observed minor decrease in absorbance.

1. Estimation of Film Thickness. The thickness of the bentonite deposit on the $\mathrm{ZnSe}$ crystal was estimated using transmission IR spectroscopy. The transmission spectra of deposited bentonite films were recorded at four different equally spaced spots along the ZnSe crystal viz. at 1, 3, 5, and $7 \mathrm{~cm}$ from one of the cut edges of the crystal. The intensity of the $v(\mathrm{Si}-\mathrm{O})$ band at $1040 \mathrm{~cm}^{-1}$ was utilized to estimate the film thickness by using an already measured absorption coefficient for Wyoming montmorillonite. Although the bentonite used here is only about $80 \%$ montmorillonite, the absorption coefficient of montmorillonite is assumed to be sufficiently good to make an estimation of the thickness of the bentonite film. The absorption coefficient is defined by eq 7 ,

$$
\alpha=\left(\frac{\ln 10}{d}\right) A_{\max }
$$

where $A_{\max }$ is the peak absorbance of the $\mathrm{Si}-\mathrm{O}$ band, and $d$ is the film thickness. ${ }^{33}$ According to Johnston et al., ${ }^{21}$ the experimentally determined absorption coefficient for the $\mathrm{Si}-\mathrm{O}$ band at about $1040 \mathrm{~cm}^{-1}$ is $3.60 \times 10^{4} \mathrm{~cm}^{-1}$ (Wyoming montmorillonite). In our transmission experiments concerning bentonite on magnetite, the absorbance at $1040 \mathrm{~cm}^{-1}$ varied between 0.0824 and 0.126 . According to eq 7, this implies that the thickness of the bentonite film varied between 53 and 79 $\mathrm{nm}$. In corresponding experiments with bentonite settling on a ZnSe crystal without magnetite particles, the settling was interrupted after $5 \mathrm{~h}$, implying a film thickness varying between 9 and $10 \mathrm{~nm}$, that is, about 10 layers of bentonite platelets. These thicknesses of the bentonite film are far below the penetration depth of the infrared radiation, which could easily be checked since a spectrum of water above the thicker bentonite film resulted in an absorbance of 0.67 for the $\mathrm{H}_{2} \mathrm{O}$ bending mode $\left(A_{\mathrm{S}}\right)$. Using a Beer's law expression for the absorbance of water and a molar absorptivity of $22.1 \mathrm{~L} \mathrm{~mol}^{-1} \mathrm{~cm}^{-1}$ for the bending mode of water at $1635 \mathrm{~cm}^{-1}, 34$ the effective penetration depth into water was calculated to be $420 \mathrm{~nm}$, taking the 13 reflections on one side of the ATR crystal into account. A Gibbs' surface excess of water at the bentonite surface was not considered since it has been shown that this quantity is negligible even if the absorptivity of interfacial water would be 10 times larger than in bulk water. ${ }^{35}$ The penetration depth for p-polarized light is considerably larger $\left(A_{\mathrm{p}}=1.36\right)$. Thus, the thickness of the bentonite deposit is clearly much less than the penetration depth, so the thin-film approximation seems reasonable.

2. FTIR-ATR Spectra of Bentonite. In the mid-infrared region, the most intense bands of dry bentonite are located in the wavenumber region $1150-900 \mathrm{~cm}^{-1}$ (Figure 3). The dominant band in this region is very strong, even if the bentonite is a thin film, due to the large Napierian absorption coefficient of $\mathrm{Si}-\mathrm{O}$ stretching vibrations. However, if the clay film is thin enough, then a water layer in contact with the clay exhibits even stronger absorptions, especially in the $\mathrm{OH}$-stretching region (not shown in Figure 3). The $\mathrm{OH}$-stretching and $\mathrm{OH}$-bending

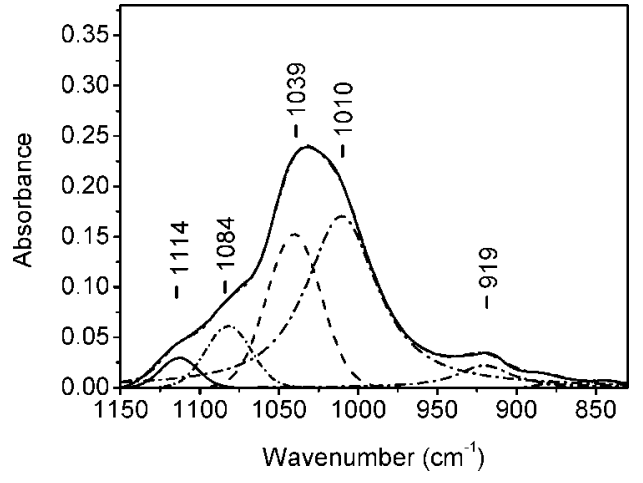

Figure 3. A p-polarized infrared spectrum of a thin $(10 \mathrm{~nm})$ film of bentonite on the internal reflection element $(\mathrm{ZnSe})$ showing the spectral region $1150-840 \mathrm{~cm}^{-1}$. The peak positions of the curve-fitted subbands (dashed lines) are indicated, except for two weak bands at 880 $\mathrm{cm}^{-1}$ and $843 \mathrm{~cm}^{-1}$. The residual root mean square (rms) error was 0.001 . The absorbance spectrum was obtained using the p-polarized single beam background spectrum of the ATR crystal.

absorptions of water on and within the bentonite film are observed at $3320 \mathrm{~cm}^{-1}$ and $1636 \mathrm{~cm}^{-1}$, respectively. In addition, the water association band due to the combination of the bending vibration and the hindered rotation of water molecules is observed at $2121 \mathrm{~cm}^{-1}$. It is well-known that the $\mathrm{OH}$ stretching vibration of water is normally located at about $3420 \mathrm{~cm}^{-1}$ in transmission spectra. However, in ATR spectra of water the peak frequency is generally observed at a lower frequency. This is due the anomalous dispersion of water, as shown in a publication by Mehmet Hancer et al. ${ }^{35}$ The absorption maximum of water varied considerably with the refractive index of the ATR element used. Thus, it was shown that internal reflection elements with refractive indices 3.43, 2.00, and 1.71 implied absorption maxima at 3370,3280 , and $3205 \mathrm{~cm}^{-1}$. Accordingly, an absorbance maximum at $3320 \mathrm{~cm}^{-1}$ would indicate that water is in contact with the $\mathrm{ZnSe}$ crystal (refractive index 2.41), or more likely, since the bentonite film is firmly attached to the magnetite particles on the $\mathrm{ZnSe}$ crystal, the position of the $\mathrm{OH}$ stretching band of water is also affected by the refractive index of the magnetite/bentonite layer at $3320 \mathrm{~cm}^{-1}$.

Upon drying the film in air, the $\mathrm{OH}$ stretching and bending bands of water eventually disappear almost completely, and in the $\mathrm{OH}$ stretching region there is only one weak band at 3620 $\mathrm{cm}^{-1}$ remaining. This band has been ascribed to the $\mathrm{OH}-$ stretching of a metal atom hydroxyl in the clay structure. ${ }^{36}$ The $\mathrm{Si}-\mathrm{O}$ stretching bands of bentonite are located to the spectral region $1200-960 \mathrm{~cm}^{-1}$. Four $v(\mathrm{Si}-\mathrm{O})$ bands are detected, viz. at $\sim 1115, \sim 1085, \sim 1040$, and $\sim 1010 \mathrm{~cm}^{-1}$. In addition, weak bands are observed below $1000 \mathrm{~cm}^{-1}$ due to bending vibration of the metal- $\mathrm{OH}$ entities within the bentonite structure. ${ }^{36}$ The peak frequencies of the latter absorption bands are at 919,880 , and $843 \mathrm{~cm}^{-1}$.

However, the most important assignments for polarized infrared measurements are the directions of the dipole moments caused by $\mathrm{Si}-\mathrm{O}$ vibrations. From previous studies, ${ }^{21,22,36-39}$ it seems clear that the band observed at $\sim 1080 \mathrm{~cm}^{-1}$ is caused by a transition dipole moment perpendicular to the (001) surface of the clay mineral and that absorption at a lower frequency, viz. at $\sim 1040 \mathrm{~cm}^{-1}$, is due to vibrations having a transition dipole moment perpendicular to the surface normal of the (001) surface (in-plane vibrations). In the present work, the assignment of the out-of-plane mode is utilized to determine the orientation of bentonite platelets settled onto the surface of the ATR crystal or onto the magnetite particles deposited on the internal reflection element $(\mathrm{ZnSe})$. 


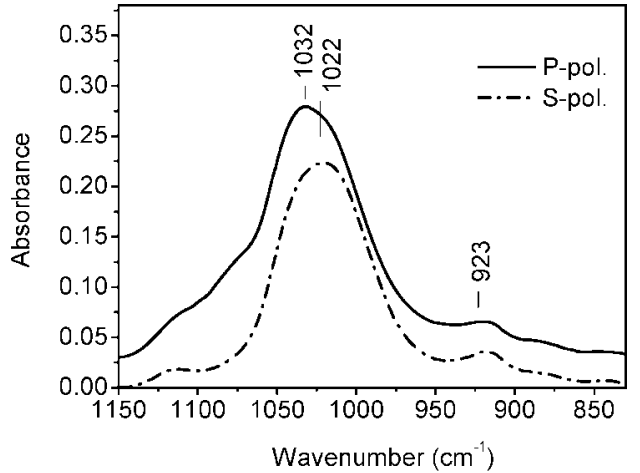

Figure 4. Infrared spectra of bentonite in the $\mathrm{Si}-\mathrm{O}$ stretching region, showing the p-polarized spectrum and the s-polarized spectrum. The absorbance spectra were obtained using single beam background spectra of the ATR crystal.

3. The Orientation of Bentonite Platelets on $\mathrm{ZnSe}$. The FTIR-ATR spectra of bentonite particles settled on the internal reflection element are sensitive to the polarization of the incident infrared beam (Figure 4). The p-polarized spectrum was obtained with the IR beam polarized parallel to the plane of incidence, while the s-polarized spectrum was obtained with the beam polarized perpendicular to the plane of incidence. The most prominent difference between the two directions of polarization is the significantly higher intensity of the band at $\sim 1080 \mathrm{~cm}^{-1}$ in the $A_{\mathrm{p}}$ spectrum. This implies a low dichroic ratio $\left(A_{\mathrm{s}} / A_{\mathrm{p}}\right)$ and consequently indicates that the bentonite platelets are oriented on the ZnSe crystal with their (001) planes preferentially parallel to the surface of the ATR crystal. A curve fit of the two band shapes in Figure 3 was accomplished by using component bands with peak frequencies at 843, 880, 919, 1010, 1039,1084 , and $1114 \mathrm{~cm}^{-1}$. The residual rms error of the curve fit was 0.001 . According to this curve fitting, the dichroic ratio of the $1082 \mathrm{~cm}^{-1}$ band was 0.26 . With the refractive index of bentonite at $1084 \mathrm{~cm}^{-1}\left(n_{2}=1.15\right),{ }^{40}$ the tilt angle of the normal to the (001) surface could be calculated using eqs $2-4$, and 6 . The tilt angle obtained $\left(\gamma=34^{\circ}\right)$ indicates that the platelets are oriented with their basal planes preferentially parallel to the ATR crystal. The dichroic ratio would have been 0.675 in order to imply randomly oriented bentonite platelets. According to IR spectra, the "dry" film contained very small amounts of water that should affect the refractive index of the film. Using $n_{2}=$ 1.248 (pure water at $1084 \mathrm{~cm}^{-1}$ ), the tilt angle became $30^{\circ}$, so the true value should be in the range between 30 and $34^{\circ}$. However, this tilt angle is considerably larger than the angle obtained by Johnston et al. ${ }^{22}$ in a similar investigation on the orientation of a monolayer of Wyoming montmorillonite on $\mathrm{ZnSe}$, viz. $12^{\circ}$. The lower value obtained for Wyoming montmorillonite may be due to the film preparation method used, where the platelets were prealigned at the water/air interface using a cationic amphiphile and subsequently compressed at various surface pressures in a Langmuir-Blodget trough before deposition on the ZeSe crystal. The settling method used here should admit more card-house-type structures in the bentonite multilayers.

4. The Orientation of Bentonite Platelets on Magnetite. Tilt angles for bentonite settled on a magnetite deposit were calculated assuming a three layer model to be valid, that is, three refractive indices are describing the components of the E-vector of the evanescent field (eqs 2-4 and 6). However, in the experiments with a magnetite film on the internal reflection element there are actually four refractive indices, since the refractive index of magnetite should be different from the corresponding index for bentonite. As can be seen from eqs $2-4$, the electric field components in $x$ and $y$ directions are independent of the refraction index of the deposited layer (magnetite and bentonite), whereas the $z$-component is strongly influenced by the value of $n_{2}$. In the dichroic ratio, $A_{\mathrm{s}}$ (absorbance measured with $y$-polarized light) is equal to $A_{\mathrm{y}}$, and $A_{\mathrm{p}}$ (measured by light polarized in the $x-z$ plane) is equal to $A_{\mathrm{x}}$ $+A_{\mathrm{z}}$. Accordingly, a precise determination of the orientation of the bentonite platelets on magnetite using the three layer model (eq 6) demands an accurate value of $n_{2}$. However, in the present study the aim was primarily to determine the preferred orientation of the platelets and not an exact value of the tilt angle.

To estimate a refractive index for the thin and porous magnetite layer, the refractive index of magnetite powder and the volume fraction of empty space between the magnetite particles are needed. However, as far as we know there is no refractive index of magnetite particles reported at $1080 \mathrm{~cm}^{-1}$. According to Handbook of Chemistry and Physics, ${ }^{41}$ the refractive index of magnetite powder is 2.42 (sodium line, 5893 $\AA$ ). The corresponding value for hematite is 3.22 . In a recent study of optical properties of thin iron oxide films on steel, ${ }^{42}$ it was shown by ellipsometric measurements that the index is 1.9 at $1200 \mathrm{~nm}$ with a declining optical dispersion $(\mathrm{d} n / \mathrm{d} \lambda)$. The corresponding value for a hematite film thermally grown on steel was 2.35. In this context it is interesting to compare the latter value with an investigation of combustion-synthesized hematite aggregates consisting of $50 \mathrm{~nm} \mathrm{Fe}_{2} \mathrm{O}_{3}$ particles, ${ }^{43}$ showing that the refractive index increased from 1.81 to 1.98 as the aggregation number increased from 3 to 7 . Experimental data were analyzed at $488 \mathrm{~nm}$.

Since reported index values for hematite are larger than the values for magnetite, it is assumed here that the refractive index of magnetite particles should not exceed 1.9. Provided the magnetite nanoparticles are close-packed spheres on the $\mathrm{ZnSe}$ crystal, it is straightforward to calculate the fraction of space occupied by the spheres, which is 0.740 . With water $(n=1.248$ at $\left.1084 \mathrm{~cm}^{-1}\right)^{34}$ in the empty space between the particles, the refractive index of the porous layer was estimated to be 1.73 , assuming a linear relationship. In case the particles are not closepacked on the ZnSe surface but have a larger fraction of empty space between them, the refractive index will be even lower. The refractive index of the wet bentonite film is also expected to be strongly influenced by the index of water since sodium bentonite is swelling clay. If the two indices are similar, then the three layer model should be valid.

Accordingly, an effective refractive index of the combined magnetite/bentonite layer should be between the indexes for "dry" bentonite (1.15) and the maximum value for a thin porous layer of magnetite particles (1.73).

Because an accurate value of $n_{2}$ was not available for the combined magnetite/bentonite layer, the dependence of the tilt angle on the refractive index $n_{2}$ was calculated (Figure 5) at the experimentally obtained dichroic ratio for a "dry" magnetite/ bentonite film $\left(A_{\mathrm{s}} / A_{\mathrm{p}}=0.30\right)$ as well as for a film covered by the aqueous suspension $\left(A_{\mathrm{s}} / A_{\mathrm{p}}=0.46\right)$.

As shown in Figure 5, the tilt angle decreases from 36 to $19^{\circ}$, upon increasing the refractive index from 1.15 to 1.7 , that is, all indices in this range indicate the platelets to be oriented with their basal planes preferentially parallel to the surface of the ATR crystal. Without having an exact value of the refractive index, the average value (1.425) was preferred, resulting in a tilt angle of $25^{\circ}$ between the surface normal of the ATR crystal and the normal to the (001) plane of the platelets. 


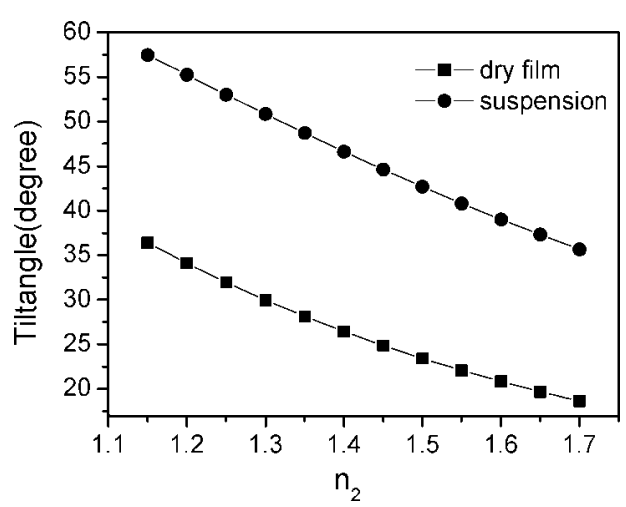

Figure 5. The calculated tilt angles vary with the refractive index of the magnetite/bentonite film $\left(n_{2}\right)$. The two curves are calculated using $A_{\mathrm{s}} / A_{\mathrm{p}}$ values at $1080 \mathrm{~cm}^{-1}$ for a dry $(0.30)$ and a wet $(0.46)$ film, as obtained from curve fitting of the measured absorption bands.

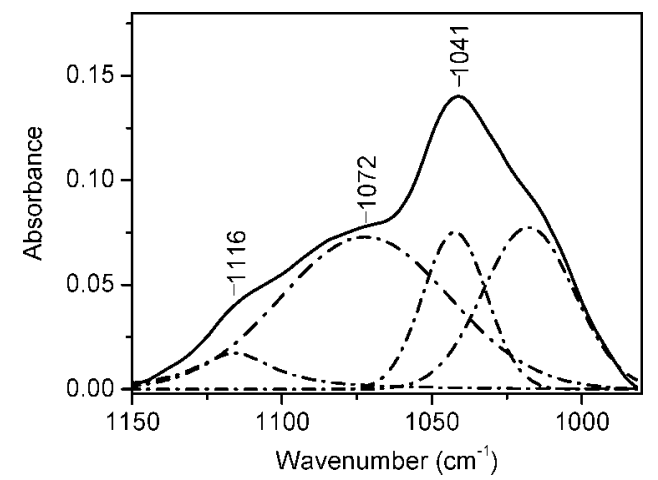

Figure 6. Curve fitting of the p-polarized $\mathrm{Si}-\mathrm{O}$ stretching region of bentonite after $4 \mathrm{~h}$ of settling. The residual rms error was 0.0004 . The component bands (dashed lines) are pure Gaussian except for the band at $1115 \mathrm{~cm}^{-1}$, which is pure Lorentzian. The single-beam background spectrum was recorded with a layer of magnetite on the ATR crystal.

The average tilt angle can be used to calculate the order parameter, $S$, frequently used for stretched polymer films and liquid crystals $\left(S=1.5 \cos ^{2} \gamma-0.5\right)$ and originally defined by R. S. Stein. ${ }^{44}$ An average tilt angle of $25^{\circ}$ gives $S=0.72$. The positively charged magnetite particles on the $\mathrm{ZnSe}$ surface is expected to increase the affinity of the surface for the first layer of clay particles, contributing to their preferential orientation. On the other hand, the magnetite layer is certainly not completely smooth and would therefore contribute to larger tilt angles since the clay particles might adapt to the topography of the underlying surface.

It is interesting to notice that the three layer model used here in the calculation of the tilt angle give reasonable values, although a four layer model, if available, would have been the natural choice. Irrespective of the refractive index in the range 1.15-1.7, the bentonite platelets are evidently oriented with their (001) surface almost parallel to the magnetite film, that is, parallel to the surface of the internal reflection element. The larger the true refractive index is, the smaller the tilt angle. Moreover, the calculations indicate that the magnetite particles contributed to an increased order of the settled bentonite particles.

The orientation of the platelets also changed with the settling time. In Figure 6, the curve fitting of the infrared absorption band after $4 \mathrm{~h}$ is shown. Before the water evaporation had resulted in a dry film, which was obtained after $7 \mathrm{~h}$, the 1080 $\mathrm{cm}^{-1}$ band in dry bentonite appears at lower frequency, viz. $1072 \mathrm{~cm}^{-1}$. The dichroic ratio of this film was $A_{\mathrm{s}} / A_{\mathrm{p}}=0.46$.
Now the medium outside the film is a water suspension in stead of air. The tilt angle calculated using eq 6 with $n_{3}=1.244$ for water at $1072 \mathrm{~cm}^{-1}$ and $n_{2}=1.425$ for the magnetite/bentonite layer showed an increase from $25^{\circ}$, as obtained for the dry film, to $45^{\circ}(S=0.25)$ for the wet film. A higher value is likely for two reasons. First, the thin bentonite film allows the evanescent wave to probe platelets still in suspension, and these platelets are likely to adopt a less-ordered orientation before they settle. This contribution is, however, expected to be small since the concentration of platelets in suspension is much lower than the concentration of already-settled platelets, although the evaporation of water will increase the concentration of the suspension. Second, a maximum of water between the platelets of the swelling clay may induce not only a broader distribution of orientations, but also a higher average tilt angle. Another reason for the increased tilt angle obtained when water is both outside and between the platelets may be that the bentonite platelets exhibit so-called card-house structures when they are about to settle on the magnetite layer. In card-house structures the edge of one platelet is interacting with the basal (001) plane of another platelet. Such an arrangement should imply larger tilt angles. One hour and a half before the film was dry, the corresponding tilt angle was reduced to $31^{\circ}(S=0.60)$. To be able to compare these angles, the same model was used for the curve fitting, that is, the band characters were either Gaussian or Lorentzian (Figure 6). Settling times closer to apparent dryness was not possible to probe because the water film was not evenly evaporated, but part of the surface along the edges of the trough began to dry. However, it seems clear that the tilt angle is lowest for the dry film and is higher if the film is covered by a water suspension of the platelets.

\section{Conclusions}

The orientation of bentonite platelets on the internal reflection element ( $\mathrm{ZnSe})$ as well as on a layer of magnetite particles deposited on the internal reflection element was analyzed with polarized FTIR-ATR spectroscopy. A thin layer of the clay particles was accomplished by settling from a diluted suspension. The dichroic ratio of the out-of-plane $\mathrm{Si}-\mathrm{O}$ stretching vibration differed significantly from the ratio for a random distribution of the platelets. For a dry bentonite film on $\mathrm{ZnSe}$, the (001) surface (basal plane) of the platelets was preferentially oriented perpendicular to the surface normal of the ATR crystal. This was also true for a "dry" bentonite film settled on a thin layer of magnetite particles deposited on the $\mathrm{ZnSe}$ crystal, but the magnetite particles contributed to an increased order (lower tilt angle). For a wet film, the tilt angle of the bentonite platelets was considerably larger indicating that the swelled bentonite layer above the $\mathrm{ZnSe}$ crystal contained platelets that tended to form so-called card-house structures at this $\mathrm{pH}$ (5.6).

Acknowledgment. This research was financially supported by funds donated by LKAB to the Hjalmar Lundbom Research Centre (HLRC) at Luleå University of Technology.

\section{References and Notes}

(1) Delville, A.; Laszlo, P. New J. Chem. 1989, 13, 481.

(2) Quirk, J. P.; Marcelja, S. Langmuir 1997, 13, 6241.

(3) Lagaly, G.; Ziesmer, S. Adv. Colloid Interf. Sci. 2002, 100, 105.

(4) Permien, T.; Lagaly, G. J. Colloid Polym. Sci. 1994, 272, 1306.

(5) Tombácz, E.; Majzik, A.; Horvát, Z. S.; Illés, E. Rom. Rep. Phys. 2006, 58 (3), 281.

(6) Rosen, M. J., Surfactants and Interfacial Phenomena, 2nd ed.; John Wiley \& Sons: New York, 1989.

(7) Eisele, T. C.; Kawatra, S. K. Miner. Process. Extr. Metall. Rev. $2003,24,1$. 
(8) Forsmo, S. P. E.; Forsmo, S-E; Björkman, Bo; Samskog, Per-Olof Powder Technol. 2008, 82, 444.

(9) Forsmo, S. P. E.; Apelquist, A. J.; Björkman, B. M. T; Samskog, P.-O. Powder Technol. 2006, 169, 147.

(10) Wang, K.; Xing, B. J. Environ. Qual. 2005, 39, 342.

(11) Evanko, R. C.; Dzombak, D. A. J. Colloid Interface Sci. 1999, 214,189

(12) Johnson, B. B.; Sjöberg, S.; Persson, P. Langmuir 2004, $20,823$.

(13) Dobson, K. D.; McQuillan, A. J. Spectrochim. Acta, Part A. 1999,

55,1395

(14) Persson, P.; Axe, K. Geochim. Cosmochim. Acta 2005, 69, 541.

(15) Hug, S. J. J. Colloid Interface Sci. 1997, 188, 415.

(16) Hug, S. J.; Sulzberger, B. Langmuir 1994, 10, 3587.

(17) Kang, S.; Xing, B. Langmuir 2007, 23, 7024.

(18) Johnston, C. T. Organic Pollutants in the Environment, Sawhey,

B., Ed.; Clay and Minerals Society: 1996; p 1.

(19) Umemura, Y; Yamagishi, A.; Schoonheydt, R.; Persoons, A.; De Schryver, F. J. Am. Chem. Soc. 2002, 124, 992.

(20) Ras, R. H. A.; Umemura, Y.; Johnston, C. T.; Yamagishi, A.; Schoonheydt, R. A. Phys. Chem. Chem. Phys. 2007, 9, 918.

(21) Johnston, C. T.; Premachandra, G. S. Langmuir 2001, 17, 3712.

(22) Ras, R. H. A.; Johnston, C. T.; Franses, E. I.; Ramaekers, R.; Maes,

G.; Foubert, P.; De Schryver, F. C.; Schoonheydt, R. A. Langmuir 2003, 19,4295

(23) Ras, R. H.; Schoonheydt, R. A.; Johnston, C. T. J. J. Phys. Chem. A 2007, 111, 8787 .

(24) Kamland, O., Olsson, S.; Nilsson, U., In Mineralogy and sealing properties of various bentonite-rich clay mineral, TR-06-30; Swedish Nuclear Fuel and Waste Management Co.: Stockholm, Sweden; 2006; p 26.

(25) Kang, Y. S.; Risbud, S.; Rabolt, J. F.; Stroeve, P. Chem. Mater. 1996, 8, 2209.

(26) Kim, D. K.; Zhang, Y.; Voit, W.; Rao, K. V.; Muhammed, M. J. Magn. Magn. Mater. 2001, 225, 30.
(27) Ahn, D. J.; Franses, E. F. J. Phys. Chem. 1992, 96, 9952.

(28) Mirabella, F. M.; Harrick, N. J. Internal Reflection Spectroscopy: Review and Supplement; Harrick Scientific Corporation: New York, 1985.

(29) Fringeli, U. P. Internal Reflection Spectroscopy. Theory and Applications. In Situ Infrared Attenuated Total Reflection Spectroscopy, Mirabella, F. M., Ed.; Marcel Dekker, New York, 1993; Vol. 15, Ch 3.

(30) Lidström, M. L.; Holmgren, A.; Forsling, W. Langmuir 2000, 16, 8129.

(31) Neivandt, D. J.; Michelle, L. G.; Michael, L. H.; Tripp, C. P. J. Phys. Chem. B 1998, 102, 5107.

(32) Holmgren, A.; Johansson, L. B.-Å.; Lindblom, G J. Phys. Chem. 1987, 91, 5298.

(33) Trchova, M.; Zemek, J.; Jurek, K. J. Appl. Phys. 1997, 82, 3519.

(34) Bertie, J. E.; Lan, Z. Appl. Spectrosc. 1996, 50, 8-1047.

(35) Hancer, M.; Sperline, R. P.; Miller, J. D. Appl. Spectrosc. 2000, $54,138$.

(36) Farmer, V. C. The Infrared Spectra of Minerals, Farmer, V. C., Ed.; Mineral Society: London, 1974.

(37) Lerot, L.; Low, P. F. Clay Clay Miner. 1976, 24, 191.

(38) Shewring, N. I. E.; Jones, T. G. J.; Maitland, G.; Yarwood, J. J. Colloid Interface Sci. 1995, 176, 308.

(39) Karakassides, M. A.; Gournis, D.; Petrides, D. Clay Miner. 1999, $34,429$.

(40) Mutschke, H.; Begeman, B.; Dorschner, J.; Guertler, J.; Gustafson, B; Henning, Th; Stognienko, R. Astron. Astrophys. 1998, 333, 188.

(41) Handbook of Chemistry and Physics, 71st ed.; Lide, D. R, Ed.; CRC Press, Inc.: Boston, Massachusetts; 1990, p 4-71.

(42) Goossens, V.; Wielant, S.; Van Gils, S.; Finsy, R.; Terryn, H. Surf. Interface Anal. 2006, 38, 489.

(43) Charalampopoulos, T. T.; Shu, G. Applies Optics. 2003, 42, 3957.

(44) Stein, R. S. J. Polym. Sci. 1958, 31, 327.

\section{JP8048923}

